\title{
A Comparative Study of Adaptive Denoising Techniques for Lightning Current Derivative Signals
}

\author{
O.Nedjah $^{1,2}$, A.M.Hussein, S. Krishnan, R. Sotudeh ${ }^{2}$ \\ ${ }^{1}$ Electrical and Computer Engineering Department, Ryerson University, \\ ${ }^{2}$ Communication and Electrical Engineering, Hertfordshire University
}

\begin{abstract}
The Lightning current data collected at the CN Tower during the last 16 years are noisy and need to be denoised for a precise analysis and for the accurate determination of the lightning waveform parameters. This paper deals with denoising the current derivative signals using adaptive denoising techniques. A new adaptive denoising approach proper to the lightning current derivative signals that was able to denoise a vast variety of lightning current derivative waveshapes is presented. The technique is based on a Divide-and-Conquer strategy. The supremacy of the new technique of denoising the lightning current derivative signals over the existing techniques is outlined for the worst case of signals where we reached a SNR of $167 \mathrm{~dB}$ while we kept $98 \%$ of the signal amplitude.
\end{abstract}

Index Terms - CN Tower, lightning current derivative, Adaptive denoising techniques

\section{INTRODUCTION}

Lightning is defined as a transient high current electric discharge. It occurs when some region of the atmosphere gains such a large charge that the electric fields associated with it can cause an electric breakdown of the air [1]. These transient high currents reaching the earth can be devastating to the modern society. They frequently cause blackouts and they can destroy or interrupt the operations of communication networks, aircrafts, spacecrafts, and electric and electronic devices. Protection from its hazards made of the lightning discharge an important area of research in many institutions from the seventies.

Lightning strikes to the Canadian National (CN) Tower have been observed since 1978. By the beginning of the summer of 1991, several measurement stations were operational to simultaneously capture the lightning current derivative at the $\mathrm{CN}$ Tower, the lightning-generated electromagnetic field, the lightning trajectory images taken from two orthogonal directions, and the return-stroke velocity [2].

When analyzing the lightning current waveforms, we are interested in accurate determination of the current waveform parameters of the signals measured at the CN Tower (current peak, the maximum current wavefront steepness, the $10-90 \%$ risetime to the current peak, and the pulse width at the $50 \%$ level of the peak) [3]. These parameters are essential in the construction of protection systems and adequate insulation for power systems, telecommunication networks and sensitive electronic devices. The extraction of lightning current waveform parameters is made difficult and less accurate by the existence of different kinds of noise in the recorded data.

The noise includes high frequencies, a DC offset which may be due to the measurement system, Loran-C frequencies around $100 \mathrm{kHz}$, and the interference of reflected currents due to structural discontinuities of the Tower [4]. After a brief description of the lightning current derivative measurement system, we will present typical current derivative signal, captured at the Tower, and define the current waveform parameters to be extracted and the effect of the noise on the extracted values of these parameters. The use of adaptive denoising techniques as the method of Frames, the Best orthogonal basis technique, the matching pursuit method, the Basis pursuit technique and the developed Divide-and-Conquer approach as noise removal tools will be emphasized, the results of their application in denoising the lightning current derivative will be discussed, and the superiority of the later technique will be brought out.

\section{CURRENT DERIVATIVE MEASUREMENT SYSTEM}

As a result to the phenomena of erection of high-rise buildings booming in the seventies, people were experiencing difficulties receiving their broadcasted TV programs. To solve the problem, The CN Tower (one of the world's tallest manmade free-standing structure with a height of $553 \mathrm{~m}$ ) was built in 1976 as a telecommunication hub. Since then, the CN Tower has been used as a broadcasting transmission facility, transmitting through its antennas, in both the UHF and VHF bands, more than 20 channels in Toronto.

Observations of lightning to the CN Tower were initiated in 1978. By the beginning of the summer of 1991, five measurement stations were operational to simultaneously capture the lightning current derivative at the $\mathrm{CN}$ Tower, the generated electric and magnetic field components (2 km north of the Tower), the lightning trajectory images taken from two orthogonal directions, and the return-stroke velocity.

The lightning current derivative (di/dt) measurement system is installed at the CN Tower. It consists of a 3-m (two 1.5 m-long sections) Rogowski sensing coil having a 40-MHz bandwidth with a sensitivity of $0.35 \mathrm{~V} /(\mathrm{A} / \mathrm{ns})$. The coil is placed at the $474-\mathrm{m}$ above ground level (AGL) and connected via a 146-m triaxial cable to a recording system located at the 372-m AGL. 
The recording system consists of a 10 ns, 10-bit, two- channel digitizer (Tektronix 710 A). It has a capacity of 128 kilobytes of memory per channel, which makes it able to register up to 8 return strokes in a lightning flash, each lasting $164 \mu \mathrm{s}$. A computer (Tektronix PEP 301) is used for the system control.

\section{LIGHTNING CURRENT DERIVATIVE WAVEFORM}

A lightning flash may contain up to several strokes. Every stroke is a transient high current electric discharge pulse. The Rogowski coil measures a voltage signal that is proportional to the lightning current derivative $(d i / d t)$. This signal, corresponding to a lightning return stroke, is registered on 16 kilobytes of memory by the recording system at a sampling frequency of $100 \mathrm{MHz}$. It forms what is called the current derivative waveform. Figure 1 presents a lightning return-stroke current derivative signal measured at the CN Tower on January 02, 1999 at 7:37 pm.

The measured current derivative waveforms at the CN Tower are corrupted by different kinds of noise, partly due to the initial function of the $\mathrm{CN}$ Tower as a transmission facility. Some of the noise is due to the measurement system itself, like the DC component. Reflected currents due to structural discontinuities of the Tower add other noise.

The captured signal frequency cannot exceed the $40 \mathrm{MHz}$ value since it is the coil's bandwidth. However, different frequencies are found in the current derivative waveform, ranging from a DC component to the $100 \mathrm{MHz}$ digitizer sampling frequency.

Furthermore, a low frequency component oscillating in the vicinity of $100 \mathrm{kHz}$ is always visible in the recorded signals. This low frequency noise component has been proved to be a result of Loran-C signals [5]. The Tower, in this case, acts as an efficient receiving antenna of Loran-C signals.

The spectrum of a current derivative waveform is shown on Fig. 3.

While analyzing a lightning current derivative signal, we are interested in determining the current waveform parameters, namely, the current peak, the maximum current wavefront steepness, the $10-90 \%$ risetime to the current peak, and the pulse width at the $50 \%$ level of the peak.

Depending on the current peak and the current wavefront steepness, the return-stroke lightning current pulse may be distinguishable, slightly exceeding the noise level, or completely embedded in the noise. This makes the extraction of the current waveform parameters difficult, inaccurate or sometimes impossible.

When the lightning signals are collected at transmission lines the first step in any analysis is the noise removal. Several methods have been tried to de-noise the lightning current derivative waveforms captured at the $\mathrm{CN}$ Tower from the associated noise as the Linear Filtering, the Fourier and the Short Fourier Transforms, the Wavelet Transform and its adaptive version [4, 68]. The adaptive techniques of denoising such as the adaptive wavelet transform, the method of Frames, the Matching Pursuit, the Best Orthogonal Basis, the Basis Pursuit method and the Divide-and-Conquer method will be described in the following section, and the results of their application in denoising the lightning current derivative will be analyzed.

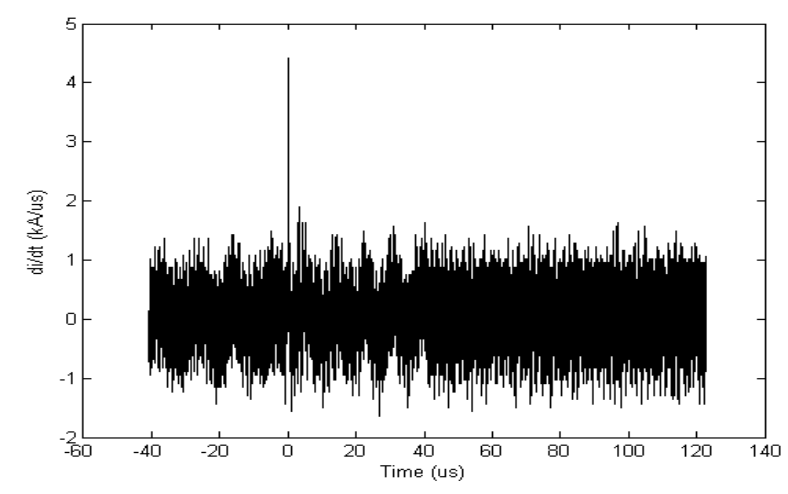

Figure 1. Measured current derivative waveform

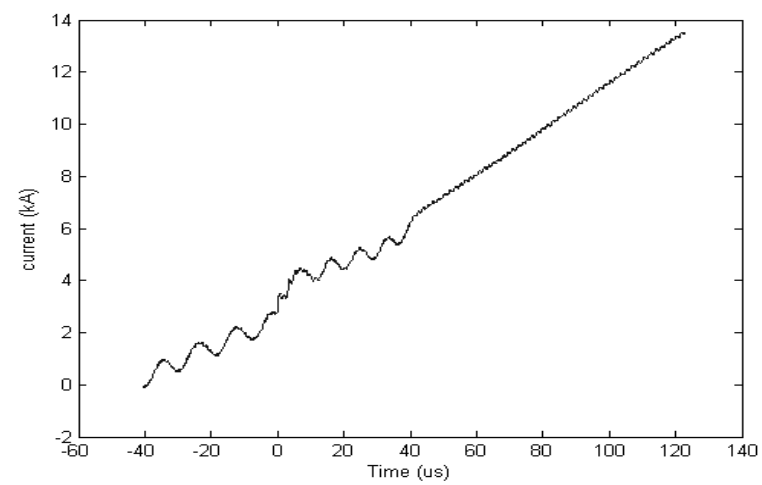

Figure 2. Current waveform

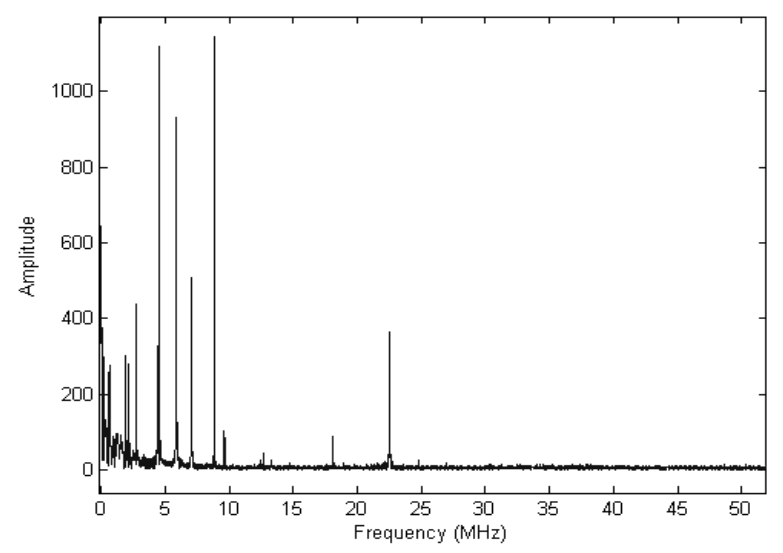

Figure 3. Current derivative spectrum

\section{ADAPTIVE DENOISING TECHNIQUES}

\subsection{Denoising Techniques}

A desired measured signal $\mathrm{s}$ is always accompanied by noise during its recording. The general recorded signal can be represented by:

$Y=s+\sigma z$

Where, $\mathrm{z}$ is supposedly a Gaussian $N(0,1)$ additive noise and $\sigma$ its level. 
The discrete signal $s=\left(s_{t}\right)_{t=0}^{n-1}$ can be approximated by the elements of a dictionary $D,\left(\phi_{\gamma}\right)_{\gamma \in \Gamma}$, where $\gamma$ is a parameter, by the expression:

$s=\sum \alpha_{\gamma_{i}} \phi_{\gamma_{i}}+R^{m}$, where $R^{m}$ is a residual.

When denoising adaptively a signal we usually represent it in a specific basis according to the adapted method (dictionary elements $\left.\left(\phi_{\gamma}\right)_{\gamma \in \Gamma}\right)$ that can be the Fourier transform, the wavelet transform, the wavelet packet transform, the cosine/sine packet transform, Gabor functions or any other function, and then consider only the components that contribute the most to the energy of the signal, or reject all parts of the signal (the residual $R^{m}$ ) that are below a specific threshold defined arbitrarily, empirically, or statistically [9-14]. The threshold is usually chosen so that it satisfies certain conditions as a minimal squared error on the estimated function:

$E\|\hat{s}-s\|_{l_{n}^{2}}^{2}$,

and a high probability that:

$|\hat{s}(t)| \leq|s(t)|, \quad \forall t$

\subsubsection{The Adaptive Wavelet Denoising}

In general, the noise associated with a signal constitutes an irregularity in the signal and can be located in the high frequencies that means in the details. This is why it can be dissociated from the signal by eliminating those details.

By removing or reducing the details selectively at desired levels, we can eliminate or reduce the effect of certain frequencies, and hence reduce the undesirable noise.

To reduce or eliminate certain details, we have to find a threshold below which the detail is eliminated or reduced. Choosing the proper threshold and the way to apply it to the details are some of the most important tasks in the denoise process. A threshold satisfying these conditions can be estimated at every level. The standard threshold $t h=\sigma \sqrt{2 \log _{10} N_{j}}$ established by Donoho is used extensively [9].

An adaptive threshold specific to the nature of the lightning current derivative signal was calculated by the following algorithm [7, 8]:

At every level of decomposition the threshold is given by:

-Find the location of the maximum value of the signal.

-Delimit a zone around the maximum value.

-Detail coefficients outside delimited zone $=$

Whole detail Coefficient - Detail coefficients of delimited zone Threshold $=$

standard deviation(Detail coefficients outside delimited zone )$* \sqrt{ }(2 * \log ($ length(Detail coefficients outside delimited zone $)))$

(4)
The adaptive denoised lightning current derivative has been estimated by evaluating the wavelet transform of the measured waveform, calculating the threshold for every level of decomposition satisfying (4) and applying it to truncate the details, and then reconstructing the waveform with the new modified details.

\subsubsection{Denoising in the Method of Frames}

Denoising in the Method of Frame (MOF) [10] is brought to minimizing the least square fit error plus a penalizing term of the expression:

$$
\min _{\alpha}\|s-\Phi \alpha\|_{2}^{2}+\lambda\|a\|_{2}^{2}
$$

With $\lambda$ is a penalizing parameter usually taken as the standard Donoho threshold equal to $\sigma \sqrt{2 \log (p)}$ where $\sigma$ is the estimated noise variance and $p$ the dictionary cardinality

This expression is generally solved by mean of linear algebra matrix inversion tools In the case of a tight frame dictionary such as the wavelet packet or the cosine packet dictionary the solution of (5) reduces to:

$$
\alpha^{+}=\frac{1}{L+\lambda} \Phi^{T} y
$$

Where $L=p / n$ is the degree of redundancy of the dictionary. This expression shows that the method of frames denoising shrinks linearly by the same amount every atom that is not orthogonal to the signal. Consequently, the reconstructed signal is just a scaled version of the original noisy observations defined by.

$$
\hat{s}=\Phi \alpha^{+}=\frac{1}{L+\lambda} y
$$

\subsubsection{Denoising in the Best Orthogonal Basis}

The tree like structure of the wavelet packet transform gives it very special properties, as certain special subcollections of the elements in this tree (dictionary elements) amount to orthogonal bases; in this way one gets a wide range of orthonormal bases. The Best orthogonal Basis (BOB) algorithm [11] picks from among these many bases a single orthogonal basis that is the "best basis".

If $\left(\mathrm{s}[\mathrm{B}]_{\mathrm{I}}\right)_{\mathrm{I}}$ denotes the vector of coefficients of a signal $s$ in the orthogonal basis $B$, and if an entropy function $E(s[B])=\Sigma_{I} e\left(s[B]_{I}\right.$ ) is defined, where $e(s)$ is a scalar function of a scalar argument, BOB solves:

$\operatorname{Min}\{E(s[B]): B$ orthogonal basis $\subset D\}$.

Once the best basis is chosen by (8), denoising comes to truncating the elements of the best basis by a specific presestablished threshold.

\subsubsection{Denoising by the Matching Pursuit Method}

In the Matching Pursuit (MP) strategy [12], the signal is decomposed in the Gaussian or Gabor functions or any other type of functions by (1). The part of the signal that contributes to the maximum energy is selected and removed from the signal. The residue $\left(R^{m}\right)$ is processed in the same way until we reach a level 
considered as the noise level which will be rejected and the signal is reconstructed back by all the components that have been selected during the process.

\subsubsection{Denoising by the Basis Pursuit Method}

In the Basis Pursuit strategy [13] the signal is decomposed in the wavelet packet, cosine/sine or any other basis. The result of decomposition is put in a matrix format and the matrix representation is optimized to count only the elements that are fundamental to represent the signal, which means the optimal basis. Hence basis pursuit comes to a matrix reduction. In this method, the problem is formulated and tackled as if it was a linear programming issue by minimizing the $l_{1}$ norm of the representation coefficients to obtain the optimum basis in which the signal can be represented by solving:

\section{$\left\{\operatorname{Min}\|\alpha\|_{1}\right.$ \\ $\{$ subject to $\Phi \alpha=s$}

Translating the denoising problem into the basis pursuit philosophy, the following will be solved:

$$
\min _{\alpha} \frac{1}{2}\|y-\Phi \alpha\|^{2}+\lambda\|\alpha\|_{1}
$$

The solution $\alpha(\lambda)$ is a function of the parameter $\lambda$, assuming the dictionary to be normalized so that $\left\|\Phi_{\gamma}\right\|_{2}=1$ for all $\gamma, \lambda$ is set to the value $\lambda_{p}=\sigma \sqrt{2 \log (p)}$, where $p$ is the cardinality of the dictionary and $\sigma$ is the noise variance.

The result of optimization that is the optimum (best) basis is searched via an optimization technique as the simplex method or the primal-dual interior barrier method to look for the basis that best contribute to the signal and reduces the noise [14,15].

After preprocessing the current derivative and its associated integrated current waveform for noise reduction as it will be clarified in the next section, the parameters of the waveforms are calculated automatically. The parameters of the current waveform consist of the first peak current, the rising time (rise from $10 \%$ to $90 \%$ of the current first peak), the decay time (decay from $90 \%$ to $10 \%$ of the first peak) and the width of the current waveform at $50 \%$ of its strength.

\subsubsection{Lightning Current Derivative Denoise by Divide-and-Conquer}

As outlined throughout the described methods a primordial knowledge or guess of the signal characteristics or statistical behavior is necessary for denoising.

By tracking the results obtained by the different denoising methods on the lightning current derivative signal we developed a strategy proper to the characteristics of the lightning current derivative and its associated current waveform obtained by its integration.

The new strategy is built upon the ideas developed in the optimization domain as divining an initial value for the optimal solution to the problem and building on it to get to the real optimum one, and it rests on the Divide-and-Conquer principle. It has been already stated and shown on the figures 1 and 3, that the lightening current derivative contains a wide range of frequencies from DC to 100 Megahertz. A first step in the pretreatment of the data will be the removal of the DC part that is due to the measurement system. We know from the Rogowski coil bandwidth that the maximum frequency that may be recorded in the lightning could be less than or equal to $40 \mathrm{MHz}$, hence the waveform is smoothed to reduce the range of high frequencies. This process reduces the number of zeros crossing in the current derivative waveform. Since the current waveform is obtained by integration, the high frequency noise is automatically removed from this waveform, which makes the search for the local minima and local maxima on the waveform much easier.

After these preliminary processes, the Divide-and-Conquer process starts based on the initial and final conditions and the Newton-Raphson optimization technique [15].

The lightning current derivative is divided into three sections; a) the time before the lightning discharge, b) the time during the lightning discharge, and c) the time after the discharge.

As initial and final conditions, it is known that there is no lightning signal before the first lightning discharge as 20 (40 or 80) $\mu$ s of signal are registered before the triggering process takes place hence the signal before this time is equalized to zero.

After the discharge there is no more peaks in the lightning current or its derivative other than the peaks reflected from the discontinuities of the tower, and the current will keep on decaying until reaching the zero value after some milliseconds, so after approximately $10 \mu$ s to $20 \mu$ s the current derivative signal can be replaced by its mean value, hence reducing more noise.

The positive and negative peaks on the current derivative waveform correspond to the maximum steepness of the current waveform respectively on ascending and descending curvatures, and the positive and negative peaks on this latter one correspond to the zero-crossing on the current derivative waveform as stated by Newton-Raphson technique. Hence the zero-crossings on the current derivative waveform together with the successive positive and negative peaks on the current waveform on the active period of the lightning current derivative waveform are tracked simultaneously by marching on the two graphs or set of data.

The Maximum current derivative peak is localized, and the first zero crossing of the waveform after it is searched, its position corresponds to the first current peak position which corresponds to the lightning channel discharge through the tower.

The maximum peak of the current waveform corresponds to the reflection from the ground. Two small peaks in between the first peak and the ground reflection peak are due to the reflections from the Observation Deck levels of the tower. Without the reflections from the discontinuities the current would have been decaying until reaching zero, this is why the peaks following the first peak that are due to the reflections at the discontinuities have to be removed.

Once the first and the second successive peaks are localized the noise due to the reflections is removed by subtraction. These successive processes reduce the noise from the lightning current derivative and its associate current tremendously.

\section{RESULTS}


The waveform of the lightning current derivative of Figure.1 represents a worst case of a lightning current derivative signal, where the current waveform is embedded in noise. The results of application of the described adaptive denoising techniques on this signal are depicted in Figures 4-9. The figures are self explanatory; they show the supremacy of the newly developed adaptive technique "Divide-and-Conquer" proper to the lightning current derivative waveforms over the other adaptive techniques.

Figure. 4 represents the result of the denoising process by the adaptive wavelet transform. In this process while the noise have been removed with a SNR passing from $-30.84 \mathrm{~dB}$ to $24.45 \mathrm{~dB}$, the current waveform was not completely denoised.

Figure.5 represents the result of denoising by the MOF method. We can notice that this method does not only reduce the signal amplitude but it doesn't remove the noise much and the SNR is reduced to $-33.71 \mathrm{~dB}$.

The BOB denoising method shown on Figure. 6 removes the noise with a SNR improving to $77.64 \mathrm{~dB}$ but it truncated the signal to less than a half of its value.

Represented on Figure.7 the MP method however, has simply removed the desired lightning current derivative signal and has chosen to denoise the Loran-C signal which is a noise for the measured lightning current derivative, so this method will be very interesting if we have to study the Loran-C signals captured by the CN Tower. This is expected from the MP method, as it chooses usually the coherent signals and fails in capturing sharp transitions.

The result of denoising by the BP method is represented on Figure.7. It shows that the method has succeeded in removing most of the noise with an improvement of the SNR to $17.80 \mathrm{~dB}$ with its amplitude left at $65 \%$ of it max value, but thinking of the Loran-C envelope making part of the signal it left it.

The results of denoise by the Divide-and-Conquer technique are displayed on Figure.8. We can see that not only has the noise been removed and the SNR improved to $167.62 \mathrm{~dB}$, but the amplitude of the signal has been preserved at $98 \%$ of its value, which proves the supremacy of this method over the other denoising techniques.
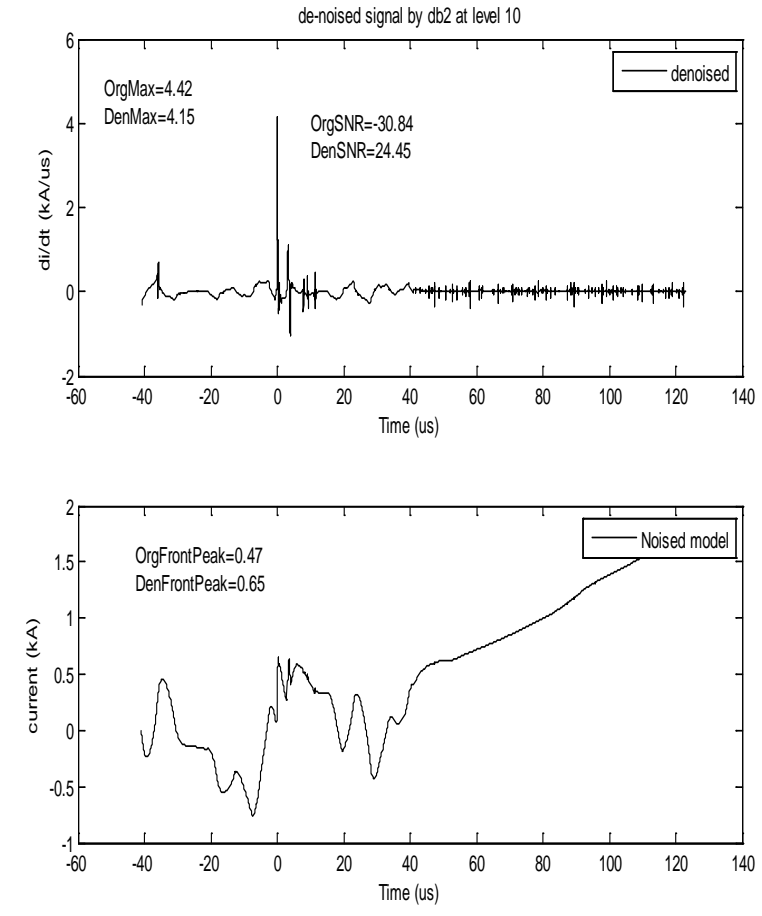

Figure 4. Noise removal by the Adaptive wavelet transform
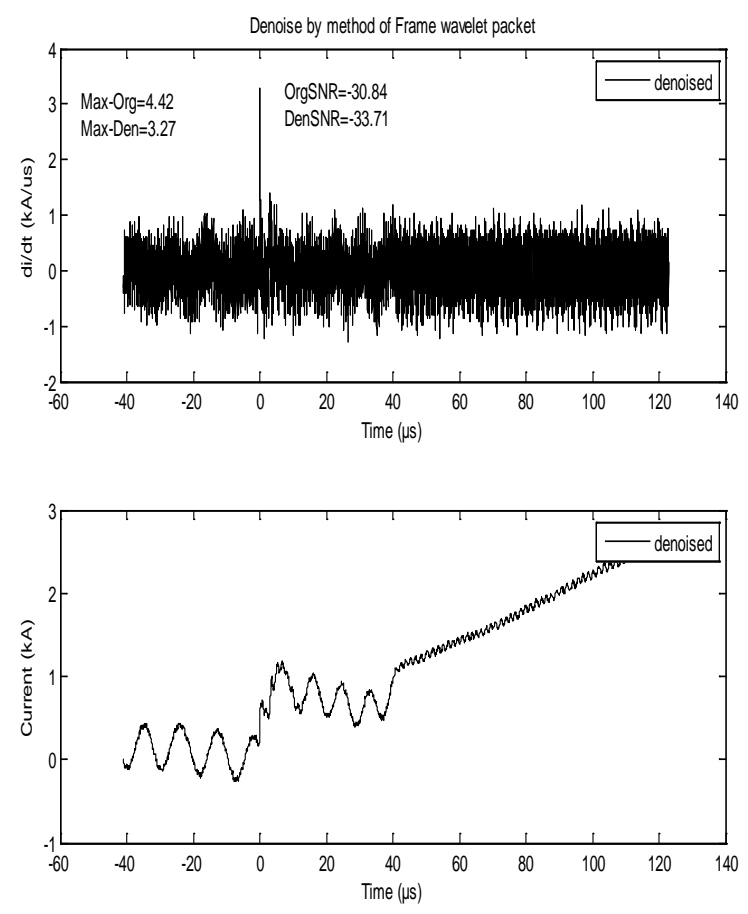

Figure 5. Noise removal by the MOF method 

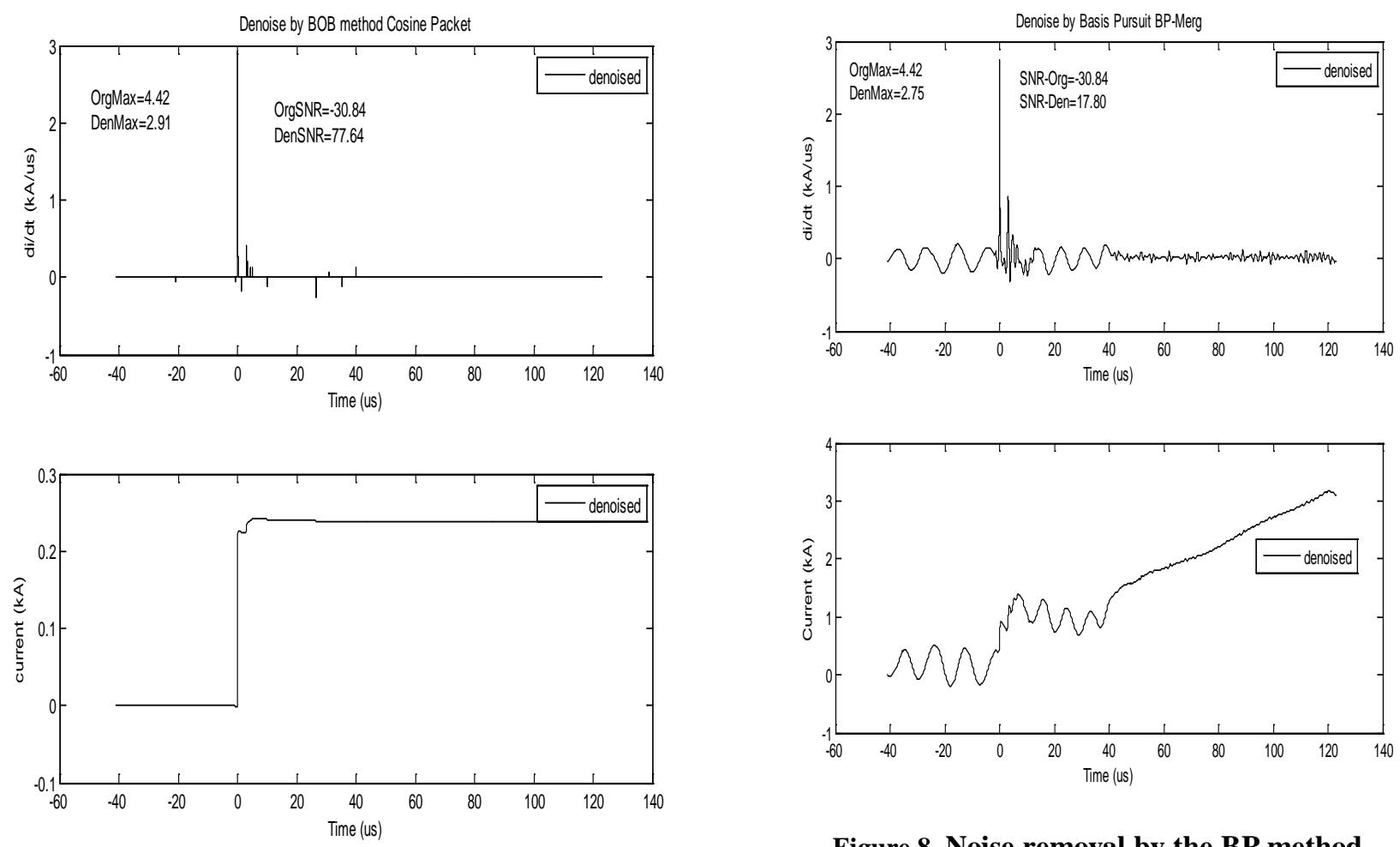

Figure 8. Noise removal by the BP method

Figure 6. Noise removal by the BOB method
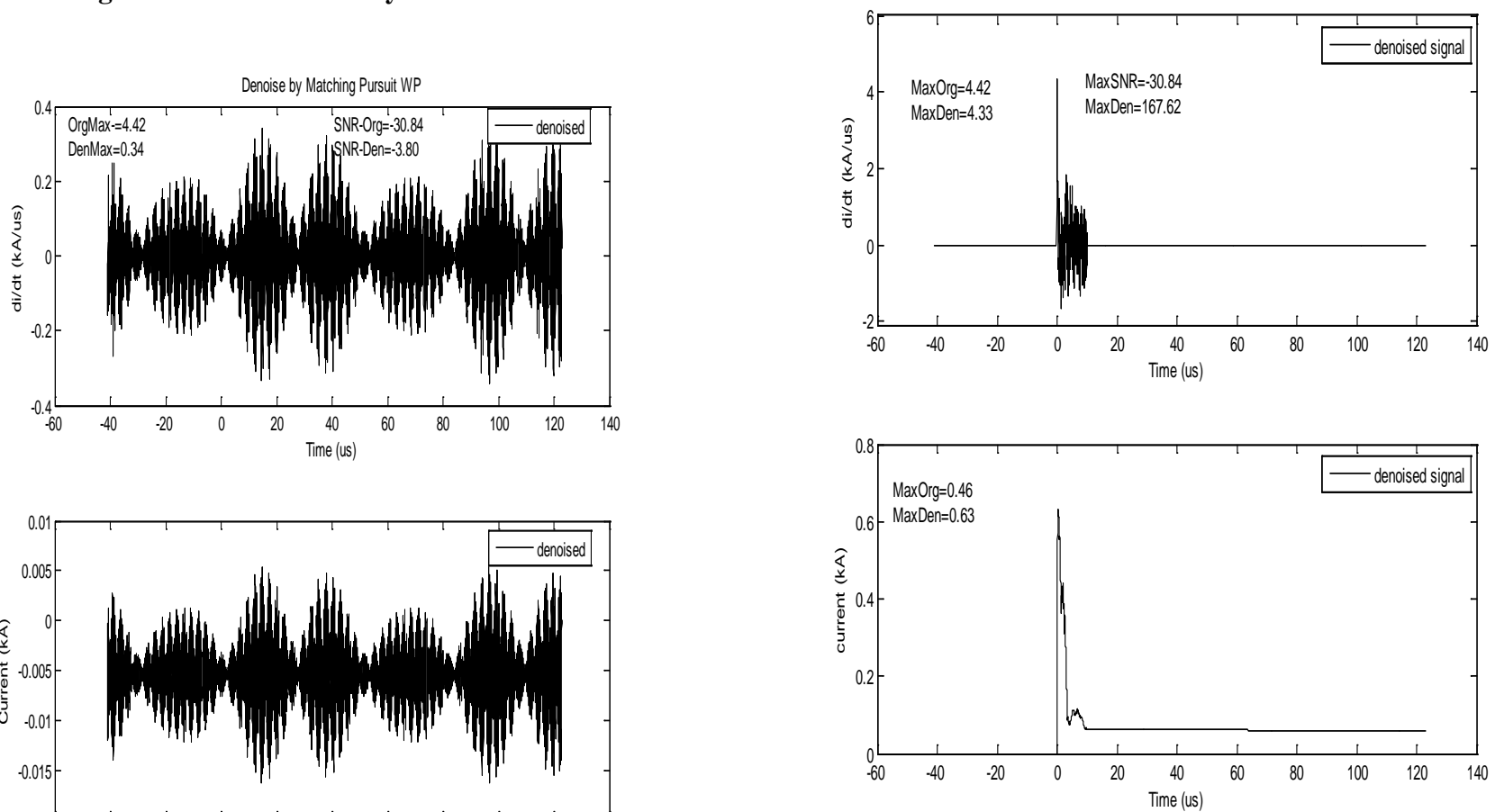

Figure 9. Noise removal by Divide-and-Conquer method

Figure 7. Noise removal by the MP method

\section{CONCLUSION}


After investigating all the ways used to overcome the problem of dependence of the denoising process on the signals on hand we have chosen the most adequate way that best tackles our problem. Inspired by the basis pursuit technique that chooses the best basis that represent a signal and removes the noise from it by optimizing its decomposition coefficients, and the marching on the graph procedure in the Newton-Raphson optimization technique to find the optimum points of a signal, we have been able to create a new method for denoising the lightning current derivative waveform and its associated current waveform. This new strategy divides the lightning current derivative according to its contents. It reduces the signal to zero on the absence of the lightning, and reduces it to its mean part at its decaying portion, and it conquers its active part and removes from it the high frequencies and the noise due to the reflections, hence reducing the noise to its minimum and facilitating the evaluation of the lightning current derivative and its integral parameters. This paper showed that for the worst case of signals where the current is diluted in the noise we reached a SNR of $167.61 \mathrm{~dB}$ while we kept $98 \%$ of the signal's amplitude. This study proved the supremacy of the new denoising technique over the existing methods for denoising the lightning current derivative waveform and its associated current waveform.

\section{REFERENCES}

[1] Martin A. Uman, "The Lightning Discharge," DoverPublication, Inc. Mineola, New York, 2001.

[2] A.M. Hussein, W. Janischewskyj, J.-S. Chang, V. Shostack, W.A. Chisholm, P. Dzurevych, and Z.-I. Kawasaki, "Simultaneous measurement of lightning parameters for strokes

to the Toronto Canadian National Tower," Journal of Geophysical Research-Atmosphere, vol.100, no. 5, pp. 88538861, May 1995.

[3] A.M. Hussein, W. Janischewskyj, M. Milewski, V. Shostak, J.S. CN

Chang and W. Chisholm, "Current waveform parameters of Tower lightning return strokes,” Journal of Electrostatics, Vol. 60, Nos. 2-4, pp. 149-162, March 2004

[4] Ying Chen, "Wavelet Analysis And Statistics Of CN Tower of Lightning Current Waveforms," M.E.Sc. Thesis, University Weston Ontario, London, Ontario, 1997

[5] P. Liatos and A.M. Hussein, "Characterization of Noise in the Lightning Current Derivative Signals Measured at the CN Tower," IEEE Transaction on Electromagnetic Compatibility, vol. 47, no. 4, pp. 986-997, November 2005.

[6] M.J. Islam and A.M. Hussein, "De-noising the CN Tower lightning current derivative signal using short term Fourier transform-based spectral subtraction," Proceedings of the $17^{\text {th }}$ International Symposium and Exhibition on Electromagnetic Compatibility, pp. 400-406, Wroclaw, Poland, June 29-July 1, 2004.

[7] Ouarda Nedjah, Ali Hussein, Reza Sotudeh, W.Janischewskyj "Wavelet Noise Removal From CN Tower Lightning Current Waveforms, ” International Signal Processing Conference Dallas, Texas, April 2003.

[8] Ouarda Nedjah, A.M. Hussein, S. Krishnan and R. Sotudeh, “CN Tower Lightning Current Derivative Heidler Model for the

Validation of Wavelet De-Noising Algorithm,” Proceedings, 18th International Wroclaw Symposium and Exhibition on Electromagnetic Compatibility, pp. 282 - 287, Wroclaw, Poland, June 28 - 30, 2006.

[9] David L. Donoho, "Nonlinear Wavelet Methods for Recovery of

Signals, Densities, and Spectra from Indirect and Noisy Data," Proceedings of Symposia in Applied Mathematics, Volume 47, 1993.

[10] Ingrid Daubechies, “Time-Frequency Localisation Operators; A Geometric Phase Space Approach” IEEE Trans. Inform. Theory, vol. 34, NO. 4, July 1988, pp. 605-612.

[11] R. R. Coifman and M. V. Wickerhauser, "Entropy-based algorithms for best-basis selection”, IEEE Trans. Inform. Theory, 38 (1992), pp. 713-718.

[12] S. Mallat and Z. Zhang, "Matching pursuit in a timefrequency dictionary,” IEEE Trans.Signal Proc., 41 (1993), pp. 33973415.

[13] S. Chen, D. Donoho, and M. Saunders, “Atomic Decomposition by Basis Pursuit,” SIAM Review, 43 (2001), pp. 129-159.

[14] Philip E.gill, Walter Murray, Dulce B. Ponceleon, M. Saunders, "Solving Reduced KKT Systems in Barrier methods for linear and Quadratic programming,” Technical report, SOL 91-7, July 1991.

[15] Greg Astfalk, Irvin Lustig, Roy Marsten, David Shanno, “The interior-point method for Linear Programming,” 